\title{
Delicious not siliceous: expanded carbohydrates as renewable separation media for column chromatography $\dagger$
}

\author{
Vitaly Budarin, James H. Clark,* Fabien E. I. Deswarte, Jeffrey J. E. Hardy, Andrew J. Hunt and \\ Francesca M. Kerton
}

Received (in Cambridge, UK) 16th February 2005, Accepted 4th April 2005

First published as an Advance Article on the web 18th April 2005

DOI: $10.1039 / \mathrm{b502330k}$

Expansion of native corn starch produces a high surface area mesoporous material capable of acting as a novel stationary phase for separating various mixtures of compounds.

Starch is one of the most abundant biopolymers on the planet and as such represents a vital renewable resource for sustainable development. ${ }^{2}$ Recently, a preparative route to novel expanded starch based materials was developed. ${ }^{3-5}$ This high pore volume, low density and high surface area biopolymer has opened new avenues of investigation in both the food and non-food industries. Applications are currently limited to encapsulation of flavoring compounds, fragrances and pharmaceuticals, and as a catalytic support. $^{3-5}$ Historically native starch has been used in chromatography, but due to its high density, low surface area and porosity its applications are limited. ${ }^{6}$ Remarkably we have discovered that expanded starch can be used as a chromatographic separation medium in the same way as silica and demonstrates comparable separation but using both less polar solvents and less material by weight. In this study, the physical properties, stability and chromatographic characteristics of this material have been investigated and evaluated against native starch and commercially available silica.

Native corn starch occurs as granules with low surface areas $\left(<1 \mathrm{~m}^{2} \mathrm{~g}^{-1}\right)$ and pore volumes, limiting its potential use as a chromatographic stationary phase. Expansion of starch significantly increases the surface area to between 100 and $180 \mathrm{~m}^{2} \mathrm{~g}^{-1}$, (Table 1) creating a mesoporous network containing few micropores as confirmed by porosimetry data (micro : mesoporous ratio (0.077)) and SEM micrographs (Table 1, Fig. 1). In contrast the

Table 1 Comparison of surface properties of starches to silica

\begin{tabular}{llllll}
\hline Physical property & Starch $^{a}$ & ES $^{b}$ & Silica & ESD & HES \\
\hline$S_{\mathrm{BET}} / \mathrm{m}^{2} \mathrm{~g}^{-1}$ & 0.6 & 119 & 458 & 0.3 & 132 \\
Adsorption isotherm type & $\mathrm{III}$ & $\mathrm{IV}$ & $\mathrm{IV}$ & $\mathrm{III}$ & $\mathrm{IV}$ \\
Microporous volume $/ \mathrm{cm}^{3} \mathrm{~g}^{-1 c}$ & - & 0.042 & 0.159 & - & 0.069 \\
Average pore diameter $/ \mathrm{nm}^{-1}$ & - & 14.2 & 6.2 & - & 17.9 \\
Total pore volume $/ \mathrm{cm}^{3} \mathrm{~g}^{-1}$ & - & 0.548 & 0.707 & - & 0.62 \\
Micro : mesoporous ratio & - & 0.077 & 0.222 & - & 0.11 \\
$E_{\mathrm{DR}} / \mathrm{kJ}$ mol ${ }^{-1}$ & - & 12.7 & 14.65 & - & 11.1 \\
$E_{\mathrm{T}}^{\mathrm{N}}$ & 0.44 & 0.68 & 0.86 & 0.46 & 0.66 \\
${ }^{a}$ Native starch has a surface area of $<1 \mathrm{~m}^{2} \mathrm{~g}^{-1}$ (type III adsorption \\
isotherm) and an $E_{\mathrm{T}}^{\mathrm{N}}$ value identical to that of Reichardt's dye \\
crystals. ${ }_{b}$ Expanded starch. ${ }^{c}$ Calculated through the Dubinin- \\
Radushkevich model. \\
\hline
\end{tabular}

$\uparrow$ This work is the subject of a patent application. See ref. 1.

*jhc1@york.ac.uk surface area of Kieselgel 60 silica gel (ex Fluka) greatly exceeds that of expanded starch, but exhibits a larger microporous distribution (micro : mesoporous ratio (0.222)). Micropores are particularly undesirable in chromatography since they give rise to size-exclusion effects or irreversible adsorption due to a high surface energy existing within the narrow pores. ${ }^{7}$ Nitrogen adsorption isotherms conducted at $77 \mathrm{~K}$ for native starch are of the type III in the IUPAC classification. Expansion of starch leads to isotherm change to type IV, comparable to that of silica.

Surface polarity measurements $\left(E_{\mathrm{T}}^{\mathrm{N}}\right)$ obtained using Reichardt's dye as an indicator, ${ }^{8}$ and surface energies $\left(E_{\mathrm{DR}}\right)$ calculated using the Dubinin-Radushkevich model both indicate that the polarity of expanded starch is lower than that of silica, but higher than that of native starch (Table 1). Elemental analysis of expanded starch showed no increase in the oxygen content compared to that of native starch. Therefore, it is proposed that the expansion of starch leads to enhanced accessibility of the hydroxyl groups on the surface. $E_{\mathrm{T}}^{\mathrm{N}}$ values of native starch were found to be identical to that of the dye crystals, indicating that no interactions take place between the surface and the dye.

In an attempt to demonstrate how the adsorbates interact with the adsorbent, the heats of adsorption for various probe molecules were investigated (Table 2). For polar protic molecules (water and methanol) the heats of adsorption increase with surface polarity from expanded starch to silica, while for pentane a decrease in the heat of adsorption was observed.

One of the drawbacks of expanded starch is its great affinity for water (as demonstrated by the high heat of adsorption (Table 2)), which can induce a collapse of the structure. When expanded starch is exposed to a saturated water vapor atmosphere at $40^{\circ} \mathrm{C}$ for $24 \mathrm{~h}$ the structure collapses and it returns to its pre-expanded surface area $\left(0-1 \mathrm{~m}^{2} \mathrm{~g}^{-1}\right)$. The resultant material (henceforth referred to as ESD) is amorphous with no visible porosity as evidenced by SEM micrographs (Fig. 1d) and porosimetry data (Table 1). Expanded starch stored for ten months under anhydrous conditions exhibited identical physical properties to that of the freshly prepared material. The SEM analysis of expanded starch stored under hexane for 10 months (henceforth referred to as HES) illustrates that the highly porous structure is retained and porosimetry data demonstrate no loss of surface area (Table 1).

The separation of ferrocene, acetylferrocene and diacetylferrocene was chosen as a model system. A mixture comprising $10 \mathrm{mg}$ of each component was loaded onto a $20 \mathrm{~cm}$ by $1 \mathrm{~cm}$ column of the desired stationary phase. The columns were successively eluted by $35 \mathrm{~mL}$ hexane, $45 \mathrm{~mL}$ hexane-acetone $(95: 5 \mathrm{v} / \mathrm{v}$ ) and $35 \mathrm{~mL}$ acetone (optimized conditions with respect 


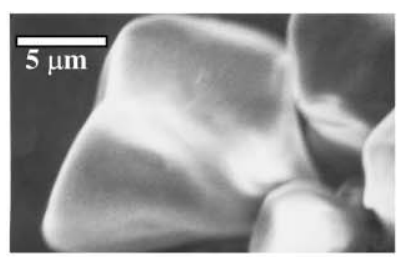

(1a) Native starch

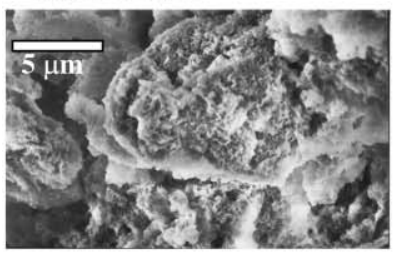

(1b) Expanded starch

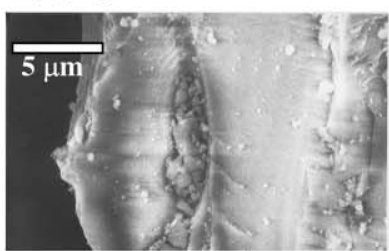

(1c) Silica K60

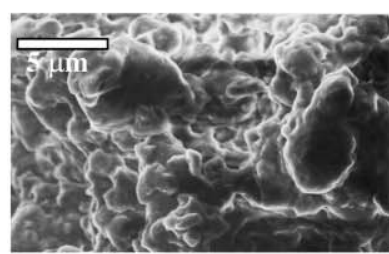

(1d) Degraded starch

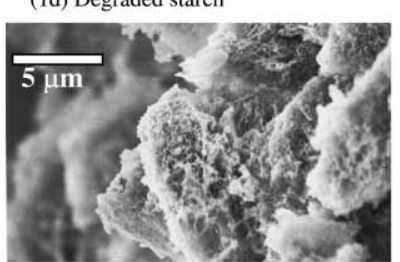

(1e) Expanded starch stored under hexane

$\square$ Ferrocene
$\square$ Acetylferrocene
$\square$ Diacetylferrocene
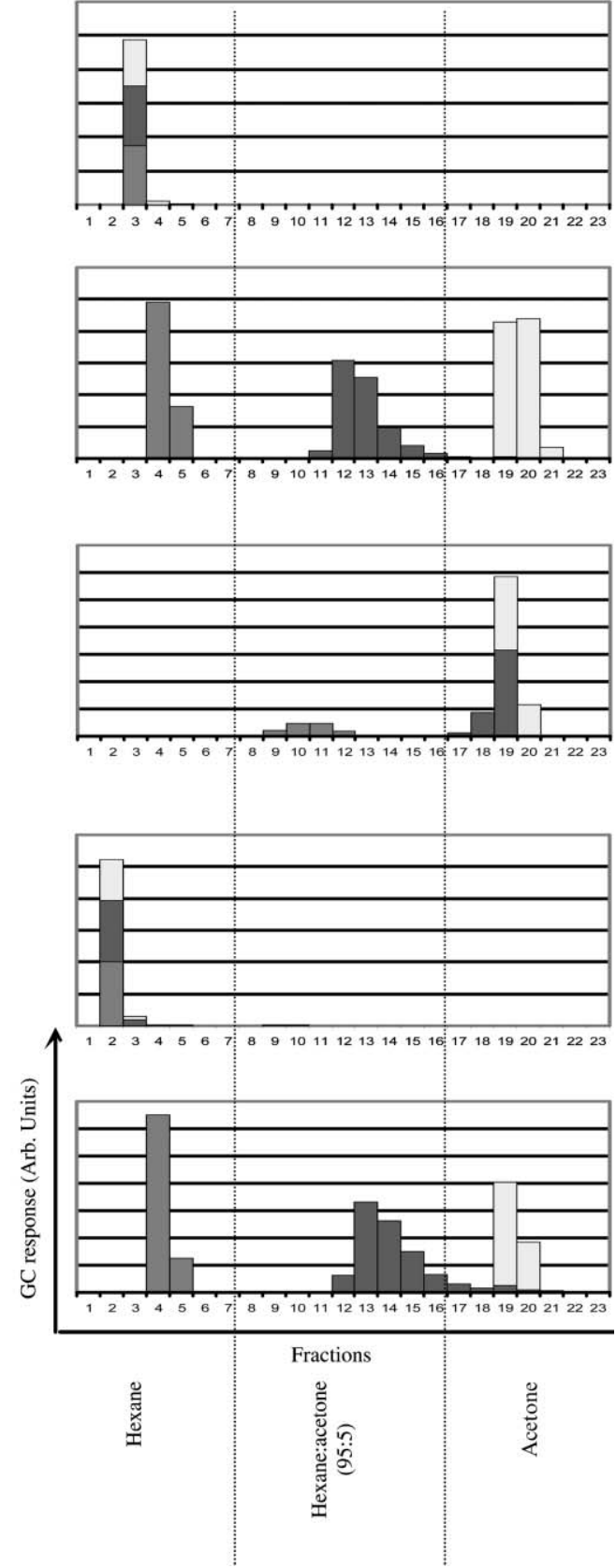

Fig. 1 SEM and separation profiles of ferrocene, acetylferrocene and diacetylferrocene over (1a) native starch, (1b) expanded starch, (1c) silica, (1d) degraded expanded starch and (1e) expanded starch stored under hexane.

Table 2 Heats of adsorption for three probe molecules for native starch, expanded starch, silica, ESD and HES

\begin{tabular}{lrrrrr}
\hline Heats of adsorption/kJ mol & & & & \\
& Starch $^{a}$ & $\mathrm{ES}^{b}$ & Silica & ESD & HES \\
\hline Water & 87.3 & 110.4 & 130.0 & 92.6 & 110.8 \\
Methanol & 95.2 & 117.7 & 134.8 & 97.5 & 104.6 \\
Pentane & 119.7 & 105.3 & 83.2 & 104.3 & 99.7
\end{tabular}

${ }^{a}$ Native starch. ${ }^{b}$ Expanded starch.

to expanded starch). Twenty-three fractions $(5 \mathrm{~mL})$ were collected and analyzed by GC.

Prior to expansion, native corn starch provided poor separation and all three components were eluted within the first $15 \mathrm{~mL}$ of hexane (Fig. 1a). In contrast the expanded starch material provided excellent separation of all three components (Fig. 1b). Separations over commercially available silica, using the model system previously described, provided no separation of the acetylferrocene and diacetylferrocene. The adsorption of components on silica was stronger than that of expanded starch, requiring more polar solvents to elute the same components (Fig. 1c); this is in correlation with results from surface polarity measurements $\left(E_{\mathrm{T}}^{\mathrm{N}}\right)$. ESD displayed chromatographic separation properties similar to those of non-expanded native starch. The preferred storage of expanded starch would be under anhydrous conditions, thereby maintaining the associated separation properties. Although the separation profile obtained from HES was similar to that of 


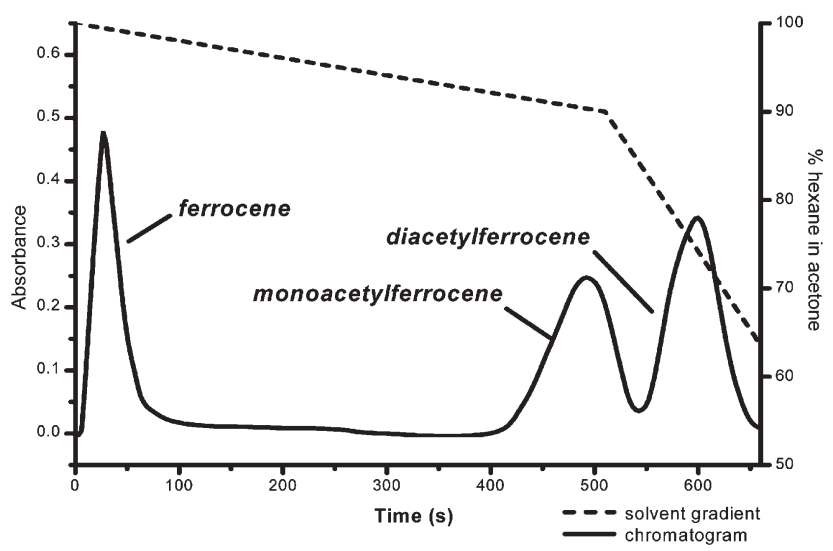

Fig. 2 High performance flash chromatogram for the separation of ferrocene, acetylferrocene and diacetylferrocene over expanded starch.

expanded starch they were not identical (Fig. 1e). An overlap in the elution of acetylferrocene and diacetylferrocene was observed, indicating a change in the chromatographic properties when stored via this method. Notable variations are also exhibited between the porous distributions of HES and the freshly prepared expanded starch material.

A comparison of the mass of material used to prepare a $20 \mathrm{~cm}$ by $1 \mathrm{~cm}$ column revealed that 3.5 times more native starch $(10.9 \mathrm{~g}(\mp 0.5 \mathrm{~g}))$ was required compared to expanded starch $(3.1 \mathrm{~g}(\mp 0.1 \mathrm{~g}))$. These values were found to be reproducible for a specific batch of expanded starch (surface area $156 \mathrm{~m}^{2} \mathrm{~g}^{-1}$ ). A significant weight reduction $(50 \%)$ was demonstrated when using columns packed with expanded starch compared to those packed with Kieselgel 60 silica gel $(6.2 \mathrm{~g}(\mp 0.1 \mathrm{~g}))$.

Expanded starch was assessed for its use in flash chromatography applications. ${ }^{9}$ The standard ferrocene mixture was separated over a $22 \mathrm{~cm}$ by $1 \mathrm{~cm}$ dry packed expanded starch column on a Horizon HPFC (Biotage), fitted with a UV detector (254 nm). A typical flash chromatogram is depicted in Fig. 2 and as demonstrated expanded starch provides excellent separation.

The expanded starch can be easily compressed into shaped forms including monoliths, which can be easily handled and are stable even in water. This may be a more appropriate form of the material for separation given the lack of well-defined particles in samples of expanded starch. The monolithic expanded starch separates mixtures of compounds with the same efficiency and at flow rates at least comparable to packed columns of particulate materials.

The reusability of expanded starch was investigated. Three separations were conducted over the same starch column and provided consistently reproducible results. Following the separations the surface area of the used starch exhibits no significant decrease (a reduction of less than 5\% was observed). Traces of water present in organic solvents had no detrimental effect on the performance of expanded starch even after repeated use.

Subsequently, other families of compounds have been investigated to assess the versatility of expanded starch in chromatography. These included lipids (sheep wool wax lanolin), functionalised aromatics, natural pigments and pharmaceuticals (caffeine and acetaminophen).

In summary, we have shown that expanded starch, akin to silica, can separate many chemical families. No separation was evident, for the compounds tested, when using native starch under identical conditions. The expanded starch separates components through adsorption, which is controlled by the surface area, surface polarity and the solvents used. It is less dense than silica and therefore a smaller mass of material can achieve the same separation. Importantly, the expanded starch material is harmless, food grade and biodegradable. Potentially, compounds with high affinities to silica, which cannot be separated on or removed from silica, could be separated on expanded starch. A more detailed understanding of the expansion process is needed to achieve control over surface area and particle size and realize the full potential of this material in separations.

We thank the EPSRC, CSL and the University of York. Additional thanks go to Dr. K. Milkowski, Mr. P. Elliott and Ms. M. Stark for their analytical support and advice.

Vitaly Budarin, James H. Clark, ${ }^{*}$ Fabien E. I. Deswarte, Jeffrey J. E. Hardy, Andrew J. Hunt and Francesca M. Kerton Centre for Clean Technology, Department of Chemistry, University of York, Heslington, York, UK YO10 5DD. E-mail: jhc1@york.ac.uk; Fax: +44 (0)1904 432705; Tel: +44 (0)1904 432559

\section{Notes and references}

1 J. H. Clark, F. E. I. Deswarte, J. J. E. Hardy, A. J. Hunt, F. M. Kerton and K. Milkowski, PCT Patent Application, PCT/GB2004/003276, 2004.

2 T. L. Barsby, A. M. Donald and P. J. Frazier, Starch, Advances in Structure and Function, RSC, London, UK, 2001; J. H. Clark and D. J. Macquarrie, Green Chemistry and Technology, Blackwell, Abingdon, UK, 2002.

3 M. Glen and D. J. Stern, US Patent, 5,958,589, 1999.

4 G. H. P. Te Wierik, J. Bergsma, A. W. Arends-Scholte, T. Boersma, A. C. Eissens and C. F. Lerk, Int. J. Pharm., 1996, 134, 27-36.

5 S. Doi, J. H. Clark, D. J. Macquarrie and K. Milkowski, Chem. Commun., 2002, 22, 2632-2633.

6 E. Soczewinski, Chem. Anal., 2003, 48, 647-666; L. S. Ettre, Chromatographia, 2000, 51, 7-17.

7 C. F. Poole, The Essence of Chromatography, Elsevier, New York, 2003.

8 D. J. Macquarrie, S. J. Tavener, G. W. Gray, P. A. Heath, J. S. Rafelt, S. I. Saulzet, J. J. E. Hardy, J. H. Clark, P. Sutra, D. Brunel, R. di Renzo and F. Fajula, New. J. Chem., 1999, 23, 725-731.

9 R. P. W. Scott, Silica Gel and Bonded Phases: Their Production, Properties and Use in LC, John Wiley \& Sons Ltd, Chichester, UK, 1993. 